\title{
Quantum formalism for systems with temporally varying discretization
}

\section{Philipp Hoehn*}

Perimeter Institute for Theoretical Physics

E-mail: phoehneperimeterinstitute.ca

\begin{abstract}
A canonical quantum formalism for discrete systems subject to a discretization changing dynamics is outlined. This framework enables one to systematically study (non-)unitarity of such dynamics, the role of canonical constraints and the fate of Dirac observables on temporally varying discretizations. It will be illustrated how the formalism can also be employed to generate a vacuum for a scalar field on an evolving lattice. Implications for the dynamics in simplicial quantum gravity models are commented on.

References

[1] P. A. Höhn, "Quantization of systems with temporally varying discretization I: Evolving Hilbert spaces" arXiv:1401.6062 [gr-qc].

[2] P. A. Höhn, "Quantization of systems with temporally varying discretization II: Local evolution moves" arXiv:1401.7731 [gr-qc].

[3] B. Dittrich and P. A. Höhn, "Constraint analysis for variational discrete systems" J.Math. Phys.54, 093505 (2013) [arXiv:1303.4294 [math-ph]].

[4] B. Dittrich, P. A. Höhn and T. Jacobson, to appear
\end{abstract}

Frontiers of Fundamental Physics 14 - FFP14,

15-18 July 2014

Aix Marseille University (AMU) Saint-Charles Campus, Marseille

${ }^{*}$ Speaker. 